\title{
DETERMINATION OF THE FACTORS THAT AFFECT MOTHERS' ATTITUDE TOWARDS IMMUNIZATION IN THE SOUTH-SOUTH REGION OF NIGERIA
}

\author{
${ }^{* 1}$ Brotobor, D., ${ }^{2}$ Nwadike, I. ${ }^{3}$ Edeawe, O. I., ${ }^{4}$ Izekor, O. C., \& ${ }^{5}$ Olowogboye, J. \\ ${ }^{1,2 \& 5}$ Department of Nursing Science, Ambrose Alli University, Ekpoma, Edo State, Nigeria. \\ ${ }^{3}$ Department of Clinical Science, Ambrose Alli University, Ekpoma, Edo State, Nigeria. \\ ${ }^{4}$ School of Post Basic Nursing, Irrua Specialist Hospital, Irrua, Nigeria. \\ "Corresponding Author Email: deliverancebrotobor@gmail.com
}

\begin{abstract}
Vaccination demand and acceptance depends on several factors that are quite broad and complex. The knowledge and attitude of a mother to childhood immunization play a key role in immunization coverage. Low immunization coverage in Nigeria is mostly attributed to poor knowledge, compliance, accessibility and inappropriate attitude among other factors. Poor compliance with full immunization is believed to be the most significant reason for low immunization coverage in the country due to socio-cultural obstacles to the acceptance of immunization. Aim: This study aims to assess the factors that determine the attitude of mothers towards immunization. Methodology: The study was carried out in Ukpenu community in Ekpoma, Edo State, Nigeria. A hundred and twenty (120) mothers were recruited into this cross-sectional descriptive study using a simple random sampling technique. Data collection was done using a structured questionnaire. The retrieved data were analyzed using the SPSS software version 21.0 and presented in frequency and percentage. Result: The study result showed that the respondents had a positive attitude towards immunization. This was evident in their immunization report. Their record revealed that most of their children were fully immunized. This was perceivable in their readiness to fully immunize their children against childhood vaccine-preventable diseases (VPDs), their willingness to recommend full immunization of children to their friends, relatives and other women, and their readiness to purchase the vaccines if they are no longer available free of charge to ensure that their children are fully immunized. It was discovered that educational status played a contributory role in the knowledge and attitude of mothers towards immunization. Conclusion: Maternal health education should be sustained to ensure continuous compliance and a positive attitude of mothers towards immunization.
\end{abstract}

Keywords: Attitude, Childhood, Factors, Immunization, Mother, Vaccination

LICENSE: This work by Open Journals Nigeria is licensed and published under the Creative Commons Attribution License 4.0 International License, which permits unrestricted use, distribution, and reproduction in any medium, provided this article is duly cited.

COPYRIGHT: The Author(s) completely retain the copyright of this published article.

OPEN ACCESS: The Author(s) approves that this article remains permanently online in the open access (OA) model.

QA: This Article is published in line with "COPE (Committee on Publication Ethics) and PIE (Publication Integrity \& Ethics)". 


\section{INTRODUCTION}

Every year more than 10 million children in low and middle-income countries die before they reach their fifth birthday (Ajala, 2017). Most of the mortality result due to lack of accessible effective interventions that could combat common and preventable childhood illnesses (Abidoye \& Odeyemi, 2016). Preventable diseases remain the most common cause of childhood mortality with an estimated 3 million deaths each year (World Health Organization [WHO], 2016). Immunization is a proven tool for controlling and eliminating life threatening infectious diseases and has been estimated to alleviate 2 to 3 million deaths each year (Wright, 2015). According to World Health Organization (2016) global vaccination coverage is holding steady, and an estimated 22 million infants worldwide are still missing out on basic vaccines. Despite the fact that immunization is proven to be the most successful and cost-effective public health intervention in reducing childhood morbidity and mortality, the global vaccination coverage has remained stalled at $86 \%$ since 2010, with no significant changes during the past years (WHO, 2016). Of the estimated 19.5 million infants that were not reached with routine immunization services worldwide in $2016,60 \%$ of them live in 10 countries including Nigeria (WHO, 2016).

According to report, only $25 \%$ of children aged $12-24$ months were fully vaccinated in Nigeria, and the neonatal, infant and under-five mortality rates remain persistently high at 37, 69 and 128 deaths per 1,000 live births respectively (National Population Commission [NPC], 2016). Furthermore, the report showed wide variations in vaccination coverage and under-5 mortality rates across Nigeria with an inverse relationship between vaccination coverage and under-5 mortalities. While the proportion of children aged 12-23 months that were fully vaccinated in Nigeria ranged from $10 \%$ in the North-West Zone, 52\% in the South-East and 38\% South-South Zone, the under-five mortality ranged from 185 deaths per 1,000 live births in the North-West Zone to 90 per 1,000 live births in the SouthWest Zone (NPC, 2016; Obinna, 2018). However, the report of the WHO and UNICEF showed that there has been record of improvement since that decade. From the report, vaccination coverage since 2015 based on their review of the 2015 National Nutrition and Health Survey (NNHS) results and preliminary results of the 2019 NNHS, suggests immunization coverage of 67 percent (WHO and UNICEF, 2020). The low immunization coverage in Nigeria plausibly explains the persistence of the transmission of the vaccine's preventable diseases in the country as the herd immunity remains low (Awosan, Ibrahim, Yunusa, Isah, Raji, et al., 2018).

\section{LITERATURE REVIEW}

It has been documented that vaccination demands and acceptance depend largely on a number of factors that are quite broad and complex (MacDonald, 2015). Furthermore, parental knowledge, attitude and practice to childhood immunization have been reported by researchers to play a key role in immunization coverage (Anand and Barnighausen, 2017). The low immunization coverage in Nigeria has been mostly attributed to poor knowledge, compliance, accessibility and inappropriate attitude among other factors; poor compliance with full immunization is believed to be the most significant reason for low immunization coverage in the country due to socio-cultural obstacles to acceptance of immunization (Oladokun, Adedekun \& Lawoyin, 2015; Adedayo, Olanrewaju, Adeyinka \& Aimahku, 2017; Ghinaia, Chris, Ibrahim \& Heidi, 2017).

More so, mother 's knowledge about immunization was found to be a predictor of full immunization in urban and rural areas of Nigeria (Odusanya et al., 2015; Black, Henry, Henderson, \& Gultiano, 2016). A study reported that 
mothers who completed at least primary level of education were 1.7 times more likely to have their children fully immunized compared to those who had no education (Caldwell, 2016). Other factors that have been found to influence compliance with full immunization of children include lack of services due to system failure, low public awareness, fear and misconceptions (Jheeta \& Newell, 2016). Several reports have shown that the uptake of immunization service depends not only on its provision but also on the knowledge and attitude of mothers, its accessibility and its adequacy in terms of manpower and material resources (Zuber et al., 2015; Anand \& Bamighausen, 2017; Uchendu, Obinna, Ibe, \& Adechie, 2018).

Some studies attributed acceptability of immunization to the kind of relationship that exists between the vaccinators and mothers, stressing on the attitude of the healthcare providers when being approached by mothers for their children vaccination (Roodpeyma et al., 2017). It has been documented that child from parents who live below the poverty level have lower immunization coverage than those whose mothers live above poverty level (Odusanya $e t$ al., 2015). Even in a population where high immunization coverage is reported; an assessment of maternal knowledge, attitude and practice on childhood immunization is believed to improve service delivery and further facilitates coverage (Oladokun et al., 2015). Hence, this study aims to assess the factors that determine the attitude of mothers towards immunization.

\section{MATERIALS AND METHODS}

A cross-sectional descriptive study design was used, with the data collected using a structured questionnaire. This study was carried out in Ukpenu community in Ekpoma, Edo State, Nigeria. The study population consists of mothers whose children were within the ages of $6-18$ months; which is the range of immunization age. After ethical approval was obtained from the relevant local government ethical committee, data was collected using a standard structured questionnaire with two (2) sections on socio-demography and attitude of mothers towards immunization. From the record, the population of the mothers attending the Ukepnu health centre was 573. After using the Taro Yamane Method for sample size calculation and considering make up for the sampling error or drop outs, 120 mothers were recruited into the study using simple random sampling technique. The 120 questionnaires were distributed, 111 were retrieved but 100 were filled completely and analyzed. The questionnaires retrieved from the study were analyzed using the SPSS software Programme version 21.0 using frequency and percentage.

\section{RESULT}

\section{SOCIO-DEMOGRAPHIC CHARACTERISTICS}

After data analysis as represented in Table 1 below, findings revealed that 90 (90\%) of the respondents were married, $6(6 \%)$ were single, while 4 (4\%) were Divorcee. The age distribution shows that $10(10 \%)$ of the respondents fell within age group 21 - 25 years, 34 (34\%) fell within age group 26-30 years, 27 (27\%) fell within age group $31-35$ years, $21(21 \%)$ fell within age group $36-40$ years and $8(8 \%)$ were within age group $41-45$ years. In terms of religion, majority of the respondents were Christians 93 (93\%), while Muslims were 7(7\%). On the education status of the respondents, 24 (24\%) had primary education, 20 (20\%) had secondary education, 45 (45\%) had tertiary education, while $11(11 \%)$ had no formal education. The respondents were drawn from different cultures; Esan 
constituted $49 \%$ of the population, Igbo, Benin, Yoruba and Hausa made up 15\%, 20\%, 12\%, and 4\% respectively of the population.

Table 1: Frequency and percentage analysis on demographic data of respondents

\begin{tabular}{llcc}
\hline \multicolumn{1}{c}{ Variables } & Categories & Frequency $(\mathbf{n}=\mathbf{1 0 0})$ & Percentage (\%) \\
\hline Age & $21-25$ years & 10 & 10 \\
& $26-30$ years & 34 & 34 \\
& $31-35$ years & 27 & 27 \\
Total & $36-40$ years & 21 & 21 \\
Marital Status & $41-45$ years & 8 & 8 \\
& & $\mathbf{1 0 0}$ & $\mathbf{1 0 0}$ \\
Total & Single & 6 & 6 \\
Religion & Married & 90 & 90 \\
& Divorce & 4 & 4 \\
Total & Christianity & $\mathbf{1 0 0}$ & $\mathbf{1 0 0}$ \\
Education & Islam & 93 & 93 \\
& & 7 & 7 \\
& & $\mathbf{1 0 0}$ & $\mathbf{1 0 0}$ \\
& Primary & 24 & 24 \\
Total & Secondary & 20 & 20 \\
Culture & Tertiary & 45 & 45 \\
& No formal & 11 & 11 \\
& & $\mathbf{1 0 0}$ & $\mathbf{1 0 0}$ \\
& & & 49 \\
& Esan & 49 & 15 \\
Total & Igbo & 20 & 20 \\
& Benin & 4 & 4 \\
& Hausa & 12 & 12 \\
& Yoruba & $\mathbf{1 0 0}$ & \\
\hline
\end{tabular}

\section{ATTITUDE OF MOTHERS TOWARDS IMMUNIZATION}

As shown in Table 2, 96\% of the respondents considered vaccination to be safe for their children and $98 \%$ considered it necessary to fully immunize their children against childhood vaccine preventable diseases. In addition, 95\% claimed that they would recommend full immunization of children to relatives and friends. It was discovered that $81 \%$ reported that most vaccines are usually unavailable and they had to return at another time to the facility in order to complete their children's immunization plan. On the determinants of the attitudes of these mothers, challenges like distance to facility (39\%) and unprofessional behavior of healthcare workers to the mothers (18\%) were identified as capable of influencing a negative change in attitude. Despite these deterrents, these women were well motivated with $86 \%$ willing to pay out of pocket for the vaccines if the free vaccine is out of stock. 
Table 2: Frequency and percentage analysis on Attitude of mothers towards Immunization

\begin{tabular}{|c|c|c|}
\hline Items & $\begin{array}{l}\text { Frequency }(\mathrm{n}= \\
\mathbf{1 0 0})\end{array}$ & $\begin{array}{l}\text { Percentage } \\
(\%)\end{array}$ \\
\hline \multicolumn{3}{|c|}{ Do you consider vaccination as safe for your children? } \\
\hline No & $\begin{array}{l}96 \\
04\end{array}$ & $\begin{array}{l}96 \\
04\end{array}$ \\
\hline \multicolumn{3}{|c|}{$\begin{array}{l}\text { Do you consider it necessary to fully immunize your child } \\
\text { against childhood vaccine preventable disease (VPDs)? } \\
\text { Yes }\end{array}$} \\
\hline No & $\begin{array}{l}98 \\
02\end{array}$ & $\begin{array}{l}98 \\
02\end{array}$ \\
\hline \multicolumn{3}{|c|}{$\begin{array}{l}\text { Would you recommend full immunization of children to } \\
\text { your friends, relatives and other women? }\end{array}$} \\
\hline Yes & 95 & 95 \\
\hline No & 05 & 05 \\
\hline \multicolumn{3}{|c|}{$\begin{array}{l}\text { Most vaccines are unavailable but I had to come back other } \\
\text { time. }\end{array}$} \\
\hline Yes & 81 & 81 \\
\hline No & 19 & 19 \\
\hline
\end{tabular}

Would you buy the vaccines if they are no longer available free to ensure that your child is fully immunized?

Yes

No

$86 \quad 86$

$14-14$

Do you consider the behaviour of the healthcare workers to influence your attitude with your immunization appointment?

Yes

$18 \quad 18$

No

Distance to the facility is the major challenge

Yes

$39 \quad 39$

No

$61 \quad 61$

\section{DISCUSSION}

Immunization is an important public health intervention strategy to reduce the morbidity and mortality associated with infectious diseases. Over two million deaths are prevented through immunization each year worldwide (WHO, 2016). Despite this, vaccine preventable diseases remain the most common cause of childhood mortality with an estimated three million deaths each year (WHO, 2016). Uptake of vaccination services is dependent not only on provision of these services but also on other factors including knowledge and attitude of mothers, attitude of health workers, 
accessibility to vaccination clinics and availability of safe needles and syringes (Anand \& Barnighusen, 2017). Mother 's characteristics have a profound influence and are the most known determinant factors of full child immunization. Hence, the study was carried out to determine the attitude of mothers towards immunization.

From this study, it is clear that demographic characteristics such as educational status played a contributory role to the attitude of mothers towards immunization. Of the 100 mothers used for the study, 89 mothers had formal education. A previous study reported that mothers who completed at least primary level of education were 1.7 times more likely to have their children fully immunized compared to those who had no education (Caldwell, 2016). This is in order with the study of Freeman, Thomason \& Bukenya (2016) that showed the relationship between mothers' education and knowledge of the diseases. Mother's knowledge on immunization can improve positive attitude that can influence full immunization of their children. Another study mentioned that maternal education is a significant determinant of child health and no other factor has such impact (Forshaw, Gerver, Gill, Cooper, Manikam \& Ward, 2017). Similarly, education empowers a woman to access relevant health services, interact effectively and assimilate information relating to prenatal care, childhood immunizations and nutritional needs (Black, et al., 2016). In concordance, Odusanya, Alufohai, Meurice \& Ahonkhai (2015) opined that the higher the mother's level of education, the better the health status of the children and family as a whole.

The results obtained showed that the respondents have positive attitude towards immunization. This was evidence in the immunization report. Their record revealed that most of their children were fully immunized. This is seen in their readiness to fully immunize their children against childhood vaccine preventable diseases (VPDs), their willingness to recommend full immunization of children to their friends, relatives and other women, and their readiness to purchase the vaccines if they are no longer available free of charge to ensure that their children is fully immunized. This study is in line with Adeleye \& Mokogwu (2016) where 99\% had positive attitude towards immunization as they stated that it is beneficial to their children. The study is also consistent with the study of Obioha, Ajala \& Matobo (2016) and Freeman, Thomason \& Bukenya (2016) who reported that mothers are interested in ensuring that their children are immunized and more so, they could encourage their co-mothers to take immunization for their children.

In this study, non-availability of vaccines on regular bases, distance to the facility and attitude of health care workers seem to have influence on the mothers. In a previous study, there was record of low respondents' satisfaction with the immunization service provision (Udonwa, Gyuse, Etokidem \& Ogaji, 2010). However, another study in Burkina Faso reported high level of satisfaction with the attitude of the health care providers (Baltussen \& Yazoume, 2006). Nonetheless, the proportion of mothers that reported to return back to the facility to ensure their children are immunized is high; their attitude might be affected if it becomes a regular occurrence. Hence, the charge nurse and the primary healthcare co-coordinator should ensure regular and prompt supply of the vaccines to ensure good compliance on the part of mothers (Wang et al., 2019).

In addition, there were a proportion of mothers who reported distance to the facility as a factor. Thirty nines (39) mothers claimed distances as a factor; which could be considered as significant. Rural-urban inequities are also another factor of immunization coverage and is certainly linked to supply-related factors, for example, accessibility to vaccination facilities and provision of childhood immunization services (Yang, Bidkhori \& Rajgopal, 2021). A 
study discovered that distance to posts as a barrier to immunization in rural areas (Adeleye \& Mokogwu, 2016). Uchendu et al. (2018) reported that about $38 \%$ of children in urban areas are reported to be more than twice as likely as rural children (16\%) to be fully vaccinated. Therefore, the charge nurse and the primary healthcare co-coordinator should ensure that strategies are developed to take the vaccines closer to the homes of the people. Regular health education on the need and benefits of full immunization of the children should be re-enforced.

\section{CONCLUSION}

Immunization plays a vital role in preventing childhood morbidity and mortality. The respondents have positive attitude towards immunization. Demographic characteristics such as educational status might play a contributory role to the knowledge and attitude of mothers towards immunization. Maternal health education should be sustained to ensure continuous compliance and positive attitude of mothers towards immunization. 


\section{REFERENCES}

Abidoye, A.O. \& Odeyemi, K.A. (2016). Knowledge, Attitude and Practice of Mothers to Childhood Immunization in Kosofe Local Government Area of Lagos State, Nigeria. Anthonio Research Center, 4(2): 66-72.

Adedayo, D., Olanrewaju, O., Adeyinka, E. \& Aimahku, C. (2017). Uptake of Childhood Immunization among Mothers of Under-Five in South Western Nigeria. The Internet Journal of Epidemiology, 7(2): 22-29.

Adeleye, O.A. \& Mokogwu, N. (2016). Determinants of Full Vaccination Status in a Rural Community with Accessible Vaccination Services in South-South Nigeria. Journal of Community Medicine and Primary Health Care, 28(1): 1-7.

Ajala, A.S. (2017). Cultural Factors Relating to Breastfeeding and their Influence on Maternal and Child Health in Ilobu, Nigeria. West African Journal of Archaeology, 32(7): 98-109.

Anand, S. \& Bamighausen, T. (2017). Health Workers and Vaccination Coverage in Developing Countries: an Econometric Analysis. Lancet, 36(8): 1277-1285.

Awosan, K. J., Ibrahim, M.T.O., Yunusa, E.U., Isah, B.A., Raji, M.O. \& Abubakar, N. (2018). Konwledge, Attitude and Compliance with Full Immunization of Children Against Vaccine Preventable Diseases among Pregnant Mothers in Sokoto, Nigeria. International Journal of Contemporary Medical Research, 5(6): 10-16.

Baltussen, R. \& Yazoume, Y. (2006). Quality of care of Modern Health Services as perceived by Users and NonUsers in Burkina Faso. International Journal Quality Health Care. 18(1): 30-34.

Black, E., Henry, H., Henderson, H. \& Gultiano, C. (2016). The Determinants of use of Maternal and Child Health Services in Metro Cuba. The Philippines Health Transition Review, 3(1): 77-89.

Caldwell, J. (2016). Education as a Factor in Mortality Decline: An Examination of Nigeria Data. Journal of Population Studies, 33(3): 395-414.

Forshaw, J., Gerver, S.M., Gill, M., Cooper, E., Manikam, L. \& Ward, H. (2017). The Global Effect of Maternal Education on Complete Childhood Vaccination: A Systematic Review and Meta-Analysis. BMC Infectious Diseases. 17(1): 1-16.

Freeman, P.A., Thomason, J.A., \& Bukenya, G.B. (2016). Factors affecting the use ofimmunization among urban settlement dwellers. New Medical Journal, 35(3): 179-185.

Ghinaia, I., Chris, W., Ibrahim, D. \& Heidi, J.L. (2017). Listening to the Rumours: What the Northern Nigeria Polio Vaccine Boycott Can Tell Us Ten Years On. Global Public Health, 8(10): 1138-1150.

Jheeta, M. \& Newell, J. (2016). Childhood Vaccination in Africa and Asia: The Effect of Parents' Knowledge and Attitude. Bull World Health Organization, 86(8): 419-420.

National Population Commission and ICF International (2016). Nigeria Demographic and Health Survey 2013. Rockville, Maryland, USA: National Population Commission and ICF International.

Obinna, C. (2018) Vaccination still Eludes 75\% of Nigerian Children-Report. Vanguard. May 27, 2018.

Obioha, E.E., Ajala, A.S., \& Matobo, T.A. (2016). Analysis of the performance of expanded programme on immunization (EPI) for four killer diseases under the military and civilian regimes in Nigeria, 1995-1999; 2000-2015. Ethno Medicine, 4(1): 43-52.

MacDonald, N.E (2015). Vaccine Hesitancy: Definition, Scope and Determinants. Vaccine. 33(34): 4161-4164. 
Odusanya, O.O., Alufohai, E.F., Meurice, P.F. \& Ahonkhai, V. (2015). Determinants of vaccination coverage in rural Nigeria. British Medical Centre Public Health, 8(2): 381-389.

Oladokun, R.E., Adedekun, B.O., \& Lawoyin, T.O. (2015). Children not receiving adequate immunization in Ibadan, Nigeria: what reasons and believes do their mothers have? Nigerian Journal of Clinical Practice, 13(6): 173178.

Roodpeyma, R., Kamali, Z., Babai, R. \& Tajik, Z. (2017). Mothers and vaccination: Knowledge, attitudes, and practice in Iran. Journal of Pediatric Infectious Diseases, 2(1): 29-34.

Uchendu, A., Obinna, J., Ibe, O. \& Adechie, K. (2018). Determinants of vaccination coverage in rural Nigeria. British Medical Center of Public Health, 381(8): 2458 - 2466.

Udonwa, N.E., Gyuse, A.N., Etokidem, A.J. and Ogaji, D.S.T. (2010). Client's View, Perception and Satisfaction with Immunization Services at Primary Health Care Facilities in Calabar, South-South Nigeria. Asian Pacific Journal of Tropical Medicine. 3(4): 298-301.

Wang, X., Peng, H., Shi,B., Jiang, D., Zhang, S. \& Chen, B. (2019) Optimizing Vaccination Strategy of a constrained Time-varying SEIR Epidemic Model. Communications in Nonlinear Science and Numerical Simulation 67: $37-48$.

World Health Organization (2016). Immunization. Available at http://www.who.int/topics/immunization/en. Accessed on 2th May 2020.

World Health Organization and UNICEF (2020) Nigeria: WHO and UNICEF estimate of immunization coverage: 2019 revision. World Health Organization and UNICEF Publication.

Wright, P.F. (2015). Global immunization: a medical perspective. Social Science and Medicine, 41(5): 609-616.

Yang, Y., Bidkhori, H. \& Rajgopal, J. (2021) Optimizing Vaccine Distribution Networks in Low- and Middle-Income Countries. Omega 99: 102197

Zuber, P.L.F., Konombo, G.K.S., Dembele-Traore, A., Millogo, J.D., Outtara, A. \& Valian, A. (2015). Mass Measles vaccination in Urban Burkina Faso, 1998. Bulletin of the world Health Organization, 79(4): 296300 . 